\title{
Utilidade da investigação rotineira de infecção fúngica pela broncoscopia em pacientes infectados ou não pelo HIV em um hospital geral, referência para SIDA
}

\author{
Usefulness of routine investigation of fungal infection through \\ bronchoscopy in patients HIV-infected or not in a \\ general hospital, reference to AIDS
}

Luiz Claudio Lazzarini-de-O liveira, Adriana A. Arantes e Maria Julieta M. Caiuby

\begin{abstract}
Resumo Avaliamos a freqüência de fungos nos materiais de broncofibroscopia em pacientes infectados ou não pelo vírus da imunodeficiência humana (HIV), quais as espécies de fungos mais encontradas e se a infecção pelo HIV alterava o perfil destes fungos. Foram revistas 1943 broncofibroscopias realizadas em hospital de referência para SIDA, no período de 1990-1995. Deste total, $47 \%$ foram realizadas em pacientes HIV positivos e 53\% em HIV negativos. Dos 908 HIV positivos, 38 (4\%) tiveram diagnóstico de micose pulmonar enquanto que dos 1035 HIV negativos, somente $4(0,2 \%)$ tiveram tal diagnóstico $(p<0,001)$. O diagnóstico de histoplasmose e criptococose predominou no grupo soropositivo para o HIV $(p<0,001)$. A paracoccidioidomicose foi diagnosticada em três pacientes não infectados pelo HIV. O estudo mostrou que, embora a positividade da pesquisa de fungos nestes materiais tenha baixo percentual de positividade, o grupo soropositivo para o HIV parece se beneficiar desta pesquisa rotineira.
\end{abstract}

Palavras-chaves: Fungos. Broncoscopia. Lavado broncoalveolar. Infecção por HIV. SIDA.

\begin{abstract}
The diagnostic yields and the spectrum of pulmonary fungal infection obtained in samples collected by fiberoptic bronchoscopy from HIV-positive and HIV-negative patients were evaluated from 1990 to 1995. A total of 1943 bronchoscopies were performed during this period, $47 \%$ in the HIV-positive group and $53 \%$ in the HIV-negative group. Of 908 HIV-positive patients, 38 (4\%) had a fungus isolated from the pulmonary sample whereas of $1035 \mathrm{HIV}$ negative patients, only $4(0.2 \%)$ had a fungus isolated. Histoplasmosis and Cryptococcosis were more frequently found in HIV-positive than in HIV-negative patients $(p<0.001)$. Paracoccidioides brasiliensis was found in only 3 patients, all of them immunocompetent. The study demonstrated that, despite the low yields, the HIV-positive group may benefit from routine screening for fungal elements in specimens obtained by fiberoptic bronchoscopy.
\end{abstract}

Key-words: Fungus. Bronchoscopy. Bronchoalveolar lavage. HIV infection. AIDS.

Serviço de Pneumologia, Setor de Endoscopia Respiratória e Serviço de Micologia do Hospital Universitário Clementino Fraga Filho da Faculdade de Medicina da Universidade Federal do Rio de Janeiro, Rio de Janeiro, RJ

Endereço para correspondência: Prof. Luiz Claudio Lazzarini de Oliveira. Secretaria de Pneumologia/HUCFF/UFRJ. Av. Brigadeiro Trompowski s/nº llha do Fundão, 21949-900 Rio de Janeiro, RJ. Telefax: 5521 290-8099

E-mail: lazzarini@openlink.com.br

Recebido para publicação em 13/7/97. 
Os fungos são microrganismos encontrados no meio ambiente e distribuídos em todo o planeta. Existem áreas, contudo, nas quais alguns fungos patogênicos têm distribuição focal, causando micoses endêmicas, tais como a histoplasmose e a coccidioidomicose nos Estados Unidos da América e a paracoccidioidomicose no Brasil5 2024 . O pulmão é a porta de entrada de muitos fungos no hospedeiro humano, tais como os agentes das micoses sistêmicas (paracoccidioidomicose, histoplasmose, coccidioidomicose, criptococose, entre outros), e de muitos agentes de micose oportunísticas (aspergilose, mucormicose, hialohifomicose, feo-hifomicose). Além dos pulmões, vários órgãos podem ser acometidos pela infecção fúngica2 ${ }^{2}$ Como o principal mecanismo de proteção do hospedeiro ao fungo é a resposta imune celular e como a infecção pelo HIV compromete esta resposta, houve um aumento na incidência das doenças fúngicas nos pacientes com síndrome da imunodeficiência adquirida (SIDA) ${ }^{14} 15$. Além de indivíduos infectados pelo HIV, outros grupos de risco podem ser atingidos como os pacientes pós-transplantados, neutropênicos e os imunossuprimidos por drogas ou outras patologias 1917.

A broncofibroscopia tem sido um método bastante utilizado para o diagnóstico de doenças pulmonares, notadamente as doenças infecciosas 679 10. Através da coleta de materiais, tais como o aspirado brônquico, o lavado broncoalveolar (LBA), a biópsia transbrônquica e a utilização de meios especiais de cultura e estudos cito e histopatológicos, pode-se freqüentemente chegar ao diagnóstico específico do agente etiológico.

O presente estudo tem por finalidade avaliar a freqüência do achado de fungos através da broncofibroscopia, as espécies de fungos mais freqüentemente encontradas e se existe uma diferença em relação a freqüência total e de cada fungo em particular nos pacientes com sorologia positiva ou não para o HIV, que foram submetidos a broncoscopia com finalidade diagnóstica. Em nosso estudo não foi avaliado o achado de pneumocistose, recentemente, reclassificado como fungo5.

\section{POPULAÇÃO E MÉTODOS}

O estudo foi realizado no Hospital Universitário Clementino Fraga Filho (HUCFF/UFRJ), de caráter terciário, referência para SIDA. No período de 1990 a 1995 foram realizadas 3.219 broncoscopias pelo Serviço de Pneumologia. Deste total, 1.943 foram realizadas com finalidade diagnóstica em que foram coletados materiais para pesquisa de fungos. As 1.276 broncofibroscopias realizadas para re-estadiamento de câncer pulmonar ou terapêuticas não foram consideradas na análise.

Foram comparados 2 grupos: 908 pacientes que apresentavam sorologia positiva para 0 HIV, feita pelo método de ELISA, complementado pelo método de imunofluorescência ou Western-Blot nos casos positivos e 1.035 pacientes cuja sorologia era negativa ou que 0 médico assistente não havia solicitado pela clínica pouco sugestiva desta infecção. Neste hospital são atendidos somente adultos.

Procedimentos. A broncofibroscopia foi realizada após administração rotineira de prémedicação com atropina e meperidina e sedação com midazolan em caso de necessidade e a coleta de material foi realizada com instilação de soro fisiológico em alíquotas de $20 \mathrm{ml}$ até um total de $100 \mathrm{ml}$ no segmento comprometido ao exame radiológico. Caso a lesão fosse difusa, era realizada coleta preferencialmente em língula ou no lobo médio. Os materiais coletados foram rotineiramente enviados aos laboratórios de microbiologia, micologia e anatomia patológica.

O diagnóstico de fungo foi firmado quando do achado na pesquisa direta, associado ao isolamento em cultivo nos meios de cultura específicos (Sabouraud, Mycobiotic, BHI, Niger e Extrato de levedura) ou pelo seu encontro nas colorações de hematoxilina-eosina, PAS e prata-metenamina realizadas no material de citologia e/ou histopatologia.

Análise estatística. A análise estatística entre os grupos foi realizada pelo teste qui-quadrado corrigido para Yates e teste exato de Fisher, quando indicado. Os resultados foram considerados significativos quando o valor de $p$ foi $<0,05$, e intervalo de confiança a $95 \%$ para as odds-ratio (OR).

\section{RESULTADOS}

No período do estudo foram realizadas 1.943 broncoscopias diagnósticas sendo 908 exames em pacientes com sorologia positiva para o HIV e 1.035 exames em pacientes com sorologia 
negativa ou não realizada. Os fungos encontrados foram: Aspergillus fumigatus, Paracoccidioides brasiliensis, Histoplasma cap sulatum e Cryptococcus neoformans. Em nossa análise não foi considerado o achado de Candida $s p$ visto que este fungo é freqüentemente encontrado nestes materiais, sendo seu achado quase que exclusivamente caracterizado como colonização e não doença, pois o seu acometimento pulmonar primário é raro4 916 .

As Figuras 1 e 2 mostram o achado de cada fungo, nos pacientes HIV positivos e HIV negativos respectivamente, nos anos do estudo. A análise estatística não mostrou um aumento significativo dos casos de infecção fúngica durante os anos analisados.

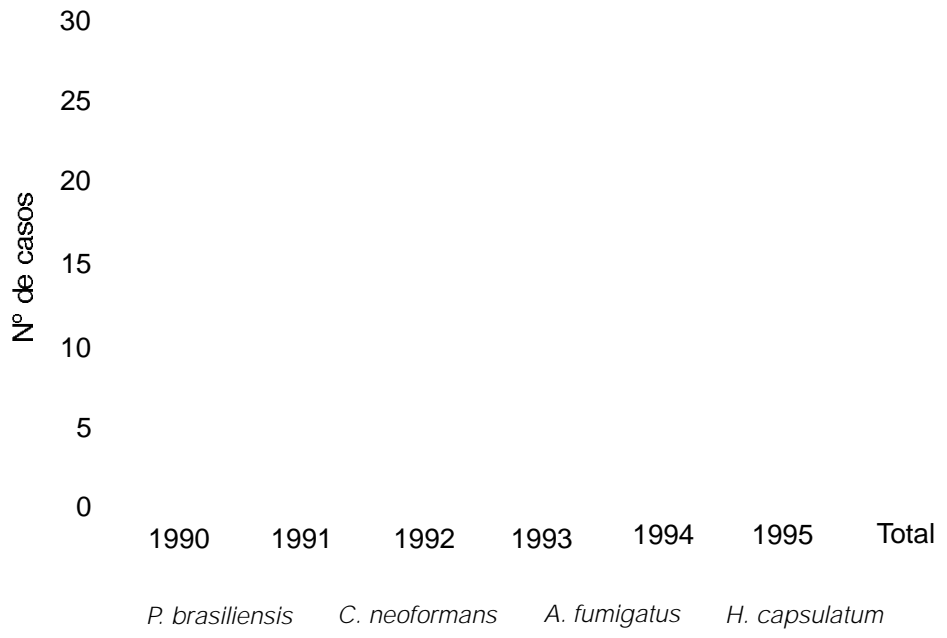

Figura 1 - Distribuição anual dos resultados da pesquisa de fundos pela broncoscopia em pacientes HIV positivos.

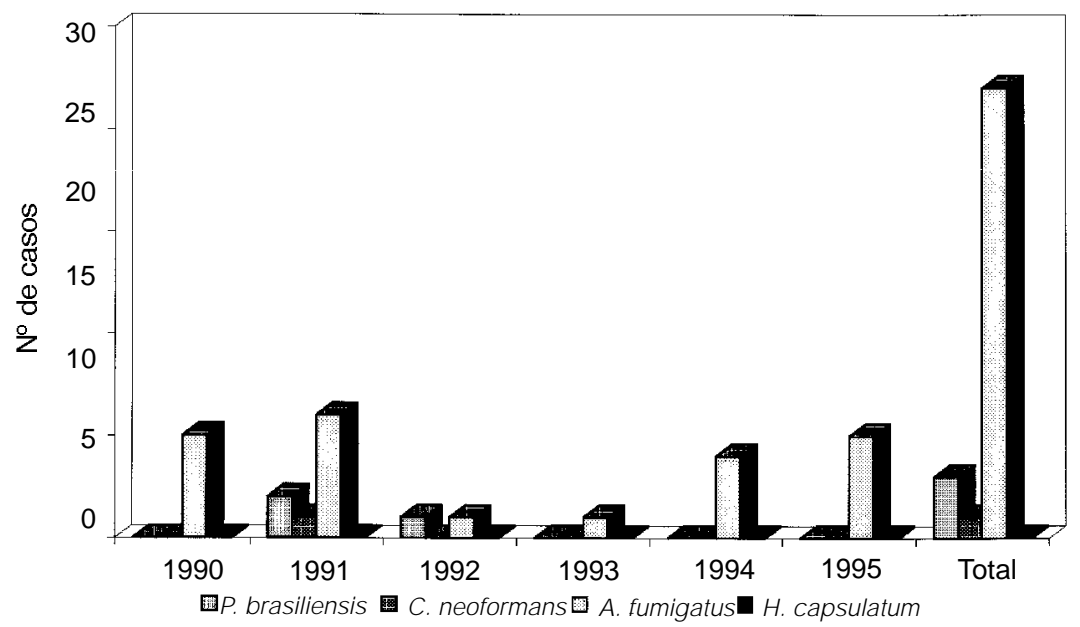

Figura 2 - Distribuição anual dos resultados da pesquisa de fundos pela broncoscopia em pacientes HIV negativos. 
A positividade geral para a pesquisa de fungos foi pequena em ambos os grupos: $4 \%$ dos pacientes do grupo com sorologia positiva para o HIV e $0,2 \%$ dos pacientes com sorologia negativa $(p<0,001)$.

Cryptococcus neoformans foi encontrado em vinte e oito (3\%) pacientes do grupo com sorologia positiva e em apenas um $(0,1 \%)$ paciente do grupo com HIV negativo [p $<0.001$ e OR 32,9 (5,4-1346)] (Tabela 1). Destes vinte e nove casos de criptococose, vinte e três $(80 \%)$ foram diagnosticados pela cultura e oito $(27,6 \%)$ foram diagnosticados pela análise cito/histopatólogica.

Histoplasma capsulatum foi encontrado em dez $(1,1 \%)$ pacientes do grupo HIV positivo e em nenhum paciente HIV negativo $(p<0,001)$.

Tabela 1 - Diagnóstico de infecção fúngica através da broncoscopia em 1.943 pacientes infectados ou não pelo vírus da imunodeficiência humana (HIV).

\begin{tabular}{lccccc}
\hline Fungo & $\begin{array}{c}\mathrm{HIV}+ \\
(\mathrm{n}=908)\end{array}$ & $\begin{array}{c}\mathrm{HIV}- \\
(\mathrm{n}=1035)\end{array}$ & p valor & OR & IC 95\% \\
\hline C. neoformans & 28 & 1 & $<0,001$ & 32,5 & $5,4-1346$ \\
H. capsulatum & 10 & 0 & $<0,001$ & & $7,6-1831$ \\
C. neoformans e H. capsulatum & 38 & 1 & $<0,001$ & 45,2 & \\
P. brasiliensis & 0 & 3 & & \\
Aspergillus fumigatus & 18 & 22 & 0,58 (NS) & & \\
\hline
\end{tabular}

NS = não significativo; OR = Odds-ratio; IC = intervalo de confiança.

Nos dez casos de histoplasmose, todos foram positivos pela cultura e somente dois foram positivos pela análise cito/histopatólogica.

Se considerarmos estes dois fungos (C. neoformans e $H$. capsulatum), suas ocorrências foram significativamente maiores no grupo de pacientes com sorologia positiva para o HIV [p < 0,001 e OR 45,2 (7,6-1831)].

Como não houve uma diferença estatísticamente significativa nos 2 grupos em relação ao encontro de Aspergillus fumigatus, e como o achado deste fungo por si só não significa necessariamente doença, revisamos os prontuários de todos os pacientes que tiveram tal diagnóstico. Arevisão teve por objetivo verificar se o paciente recebeu tratamento antifúngico para sua doença pulmonar. Dos 40 pacientes, foi possível revisar os prontuários de 36 e somente em um paciente (grupo HIV positivo) este resultado foi valorizado. Este paciente evoluiu para sepses e óbito em vigência de terapia antimicrobiana e antifúngica, porém, não foi possível obter resultados de necrópsia. Os outros 35 pacientes não receberam qualquer tratamento específico e não tiveram em seus prontuários qualquer registro de piora de doença pulmonar atribuível a este fungo. Na maioria dos pacientes não se obteve diagnóstico etiológico da doença pulmonar e em dois casos foi diagnosticado micetoma (bola fúngica) por exames radiológicos. Em 4 pacientes não foi feito acompanhamento, não sendo possível avaliar sua evolução clínica.

Em nosso estudo foram encontrados somente três casos de paracoccidioidomicose, todos no grupo com sorologia negativa para o HIV. Destes, dois foram encontrados em cultura e um na patologia.

\section{DISCUSSÃO}

A imunidade celular é o principal mecanismo de resistência do hospedeiro à infecção fúngica, sendo exatamente esta imunidade que é comprometida pela infecção pelo HIV. Embora o vírus altere de forma mais consistente a função do linfócito T CD4+ (T auxiliar), este comprometimento atinge também os linfócitos $B$, os macrófagos/monócitos e os neutrófilos, assim como a expressão de várias citocinas, causando uma profunda imunodepressão e predispondo-os a um risco aumentado de infecções12 132223.

Desde o aparecimento da SIDA, houve um aumento do número de casos de doenças fúngicas, acometendo praticamente todos os órgãos, porém com acentuada preferência pelos pulmões, trato digestivo e sistema nervoso central. O fungo mais freqüentemente visto neste grupo de pacientes é Candida $s p$, porém, o principal agente causador de patologia pulmonar é o 
Cryptococcus neoformans, descrito em aproximadamente $7 \%$ dos casos ${ }^{11}$. De fato, a SIDA é considerada o principal fator de risco para as formas disseminadas de criptococose2. Clinicamente, a maioria dos pacientes com criptococose apresenta-se com meningite, contudo cerca de um terço a metade dos casos tornam-se disseminados, sendo freqüente o achado do fungo em materiais de broncoscopia em estágios avançados da doença19. Em nosso estudo, a maioria dos pacientes com $C$. neoformans tinha diagnóstico concomitante ou prévio de meningite criptocócica.

$O$ achado de fungo nos materiais de broncoscopia foi incomum, não tendo ultrapassado $4 \%$ de positividade nos pacientes HIV positivos e $0,2 \%$ nos pacientes HIV negativos, quando pesquisado de uma forma rotineira. Estes resultados estão de acordo com uma grande série que mostrou que os fungos causam menos de $1 \%$ das infecções pulmonares nos pacientes com SIDA10. No nosso estudo, a maioria dos fungos foi identificada pela cultura, e menos freqüentemente pelo exame citopatológico, resultado semelhante ao referido por Baughman, que mostrou uma maior sensibilidade da cultura em relação ao exame citopatológico6. 0 mesmo grupo, analisando o lavado brônquico e o lavado broncoalveolar, citam uma positividade de $34 \%$ para a pesquisa direta e $85 \%$ para a cultura, concluindo que ambos os materiais tiveram resultados complementares ${ }^{7}$. Neste último estudo, entre as 2.524 broncoscopias realizadas no período de 6 anos, foram encontrados $41(1,6 \%)$ casos de infecções fúngicas assim distribuídas: 25 casos de H. capsulatum, 11 de $C$. neoformans, 4 de Blastomyces dermatitidis e 1 de Coccidioides immitis. Os autores, contudo, não diferenciam quais casos ocorreram em pacientes soropositivos para o HIV, afirmando que $53 \%$ dos casos ocorreu em pacientes imunosuprimidos, principalmente pela SIDA.

Clarke et al9 em recente estudo sobre o valor diagnóstico do LBA nos pacientes HIV positivos mostraram que em 947 broncoscopias foram encontrados elementos fúngicos em 83 $(8,7 \%)$ episódios assim distribuídos: Candida $s p$ em 67, Aspergillus sp em 9, C. neoformans em 3 e $H$. capsulatum em 2 . O estudo não cita se os casos de Aspergillus sp foram considerados contaminação ou não, porém, enfatiza a raridade do encontro de infecções fúngicas e o pouco valor diagnóstico do achado de Candida $s p$ nestes materiais. Estes resultados também estão de acordo com os nossos, embora tenhamos um número proporcionalmente maior de $C$. neoformans e $H$. capsulatum em nosso material.

A histoplasmose clinicamente significativa é incomum em indivíduos normais, porém são descritos em 5 a 27\% dos pacientes HIV positivos, dependendo da região geográfica3 21 . Estudos preliminares sugerem que a broncoscopia é um método sensível para este diagnóstico? 2 Em nosso material, todos os casos de histoplasmose foram diagnosticados pela cultura e somente dois foram diagnosticados pela patologia.

A aspergilose era inicialmente colocada como uma infecção definidora de SIDA porém a reunião dos CDC (Centers for Disease Control, USA) de 1987 retirou-a da definição pois o número de pacientes infectados e relatados era pequeno8. Aaspergilose invasiva não depende da imunidade mediada por células e sim da depleção de neutrófilos e monócitos/macrófagos. O diagnóstico requer a documentação de invasão tissular em materiais de biópsia ou o encontro de fungos em outros sítios estéreis. O achado de Aspergillus $s p$ nas secreções respiratórias pode significar colonização e não infecção2 9 16, logo o seu achado nestes materiais é insufuciente para diagnosticar doença. $O$ encontro, contudo, do fungo em uma doença pulmonar compatível, sem outra causa aparente, levanta a suspeita dele ser patogênico2. Segundo Hesse et al18, o valor do diagnóstico broncoscópico na aspergilose mostrou uma sensibilidade do LBAem $33 \%$ e do lavado brônquico em $50 \%$ dos casos em que foi confirmado doença pulmonar por Aspergillus sp. Tem sido descrito que o LBA tem uma alta taxa de falsos-negativos no diagnóstico de aspergilose pulmonar invasiva em pacientes com neoplasias hematológicas ${ }^{1}$. É descrito também traqueobronquite por Aspergillus sp em pacientes com SIDA, apresentando-se como lesões em placas ou ulcerativas podendo ser rapidamente progressiva e fatal em aproximadamente $40 \%$ dos casos 10 . Neste estudo, dos 4 pacientes com diagnóstico de traqueobronquite por Aspergillus $s p, 1$ estava neutropênico e os outros 3 estavam em uso de corticosteróide. De fato, mielossupressão é o maior fator de risco para o desenvolvimento de aspergilose pulmonar invasiva ${ }^{1}$. Atualmente, com o aumento na sobrevida dos pacientes HIV positivos, ocorrerá uma maior chance de desenvolvimento de neutropenia secundária à própria doença ou ao tratamento e, assim, a infecção por Aspergillus sp pode vir a tornar-se cada vez mais importante neste grupo de pacientes. 
Conclusões. A infecção pelo vírus HIV tem causado um aumento nas incidência das infecções oportunísticas, dentre as quais as doenças fúngicas, devido ao comprometimento da imunidade celular causada pelo vírus. Os fungos mais freqüentes nestes pacientes são o Histoplasma capsulatum e o Cryptococcus neoformans. Embora o Paracoccidioides brasiliensis seja o fungo causador da micose sistêmica mais prevalente no Brasil, não encontramos um aumento significativo do número de casos nos materiais colhidos pela broncoscopia. Mesmo considerando a baixa positividade da pesquisa de fungos nerste materiais, alguns grupos, particularmente os imunodeprimidos por drogas ou infecções (principalmente causado pelo vírus HIV), parecem se beneficiar da sua pesquisa rotineira. Consideramos, contudo, desnecessária a pesquisa rotineira de fungos nos materiais colhidos pela broncoscopia nos pacientes imunocompetentes pela raridade de seu achado, a qual deverá ser pedida somente em casos suspeitos de doença fúngica.

\section{AGRADECIMENTOS}

Agradecemos ao Prof. Afrânio Kritski pela revisão e sugestões, aos Drs. Luiz Augusto Alves Vianna e Wilza Claudia dos Anjos pelo auxílio na coleta dos dados e a Sra. Rosângela A.M. Nóe pela análise estatística deste trabalho.

\section{REFERÊNCIAS BIBLIOGRÁFICAS}

1. Albeida SM, Cassileth PA, Gerson SL, Miller WT, Talbot $\mathrm{GH}$. Role of fiberoptic bronchoscopy in the diagnosis of invasive pulmonary aspergillosis with acute leukemia. American Journal of Medicine 76:1027-1934, 1984.

2. American Thoracic Society. Fungal infection in HIVInfected person. American Journal of Respiratory and Critical Care Medicine 152:816-822, 1995.

3. Aranda CP, Salzman SH, Smith RL. Histoplasmosis in patients with risk factors for the acquired immunodeficiency syndrome(AIDS). American Journal of Respiratory and Critical Care Medicine 135:A171, 1986.

4. Basti J, Kamholz SL, Malabonga VM. Utility of bronchoscopic sampling techniques for cryptococcal disease in AIDS. Chest 99:370-372, 1991.

5. Batista L, Martinez R, Negroni R, Restrepo AM, Silva MV, Yasuda MAS. Doenças Fúngicas. In: Veronesi R, Focaccia R (eds) Tratado de Infectologia. Editora Ateneu p. 1058-1129,1997.

6. Baughman RP. Use of bronchoscopy in the diagnosis of infection in the immunocompromised host. Thorax 49:37, 1994.

7. Baughman RP, Dohn MN, Frame PT, Loudon RG. Bronchoscopy with bronchoalveolar lavage in tuberculosis and fungal infections. Chest 99:92-97, 1991.

8. Centers for Diseases Control. 1987. Revision of the CDC Surveillance definition for acquired immunodeficiency syndrome. Morbility and Mortality Weekly Report 36:1S15S, 1987.

9. Clarke J, Coker RJ, Evans DJ, Mitchell DM, Moss, Nieman R, Robinson DS, Shaw RJ, Taylor IK, Veale D. Pulmonary complications of HIV disease: 10 year retrospective evaluation of yields from bronchoalveola lavage, 1983-93. Thorax 50:12401245, 1995.
10. Covington DC, Deresinski SC, Follansbee SE, Kemper CA, Stevens AS. Ulcerative and plate-like tracheobronchitis due to infection with aspergillus in patients with AIDS. Clinical of Infectious Disease 17:344352, 1993.

11. De Vita VTBroder S, Fauci AS, Kovack JA, Chabner BA. Developmental therapeutics and the acquired immunodeficiency syndrome. Annals of Internal Medicine 106:568-581, 1987.

12. Ellis M, Galant S, Gupta S. Hakim S, Vande Vem C, Toy C, Cairo MS. Impaired neutrophil function in patients with AIDS or AIDS-related complex:a comprehensive evaluation. Journal of Infectious Disease 158:12681276, 1988.

13. Fauci AS. Immunologic abnormalities in the acquired immunodeficiency syndrome (AIDS). Clinical Research 32:491-499, 1984.

14. Fels AOS. Bacterial and fungal pneumonias. Clinics in Chest Medicine 9:449-457, 1988.

15. Felton CP, Garay SM, Murray JF. Pulmonary complications of the acquired immunodeficiency syndrome. Report of a National Heart, Lung and Blood Institute workshop. New England Journal of Medicine 310:1682-1688, 1984.

16. George S, Scott D. Fungal infection. In: Murray J (ed) Textbook of respiratory disease. $2^{\text {nd }}$ edition. Sauders. $p$. 1161-1200, 1994.

17. Hendricker CM, Mc Cubbin MM, Trigg ME, Wagener JS. Bronchoscopy with bronchoalveolar lavage in the evaluation of pulmonary complications of bone marrow transplantation in childreen. Pedriatric Pulmonology 12:43-47, 1992.

18. Hesse M, Roos N, Schulter R, Vandelco J, Vooneiff M, Zullsdorf M. Pulmonary aspergillosis:Early diagnosis improves survival. Respiration 62:341-347, 1995. 
19. Holzman RS, Loure E, Zuger A. Cryptococcal disease in patients with the acquired immunodeficiency syndrome. Annals of Internal Medicine 104:234, 1986.

20. Londero AT, Mello I. Paracoccidioidomicose. Jornal Brasileiro de Medicina 55:96-115, 1988.

21. Londero AT, Wanke B. Histoplasmose Capsulata. Jornal Brasileiro de Medicina 55:94-109, 1988.

22. Masur H, Murray HW, Roberts RB, Rubin BY. Impaired production of lymphokines and immune (gamme) interferon in the acquired immunodeficiency syndrome. New England Journal of Medicine 310:883-889, 1984.

23. Murray HW, Gellene RA, Libby DM, Rothermel CD, Rubin BY. Activation of tissue macrophages from AIDS patients: in vitro response of AIDS alveolar macrophages to lymphokines and interferon-gama. Journal of Immunology 135:2374-2377, 1985.

24. Nogueira SA, Pedrosa PN. Paracoccidioidomicose. ARS Curandi 17:96-100, 1984. 